\title{
Narrative review of prostate cancer grading systems: will the Gleason scores be replaced by the Grade Groups?
}

\author{
Rodolfo Montironi ${ }^{1}$, Liang Cheng ${ }^{2}$, Alessia Cimadamore ${ }^{1}$, Roberta Mazzucchelli ${ }^{1}$, Marina Scarpelli ${ }^{1}$, \\ Matteo Santoni ${ }^{3}$, Francesco Massari ${ }^{4}$, Antonio Lopez-Beltran ${ }^{5}$ \\ ${ }^{1}$ Section of Pathological Anatomy, Polytechnic University of the Marche Region, School of Medicine, United Hospitals, Ancona, Italy; ${ }^{2}$ Department \\ of Pathology and Laboratory Medicine, Indiana University School of Medicine, Indianapolis, IN, USA; ${ }^{3}$ Oncology Unit, Macerata Hospital, \\ Macerata, Italy; ${ }^{4}$ Division of Oncology, S. Orsola-Malpighi Hospital, Bologna, Italy; ${ }^{5}$ Department of Surgery, Cordoba University Medical School, \\ Cordoba, Spain \\ Contributions: (I) Conception and design: All authors; (II) Administrative support: None; (III) Provision of study materials or patients: None; (IV) \\ Collection and assembly of data: R Montironi, A Cimadamore, R Mazzucchelli; (V) Data analysis and interpretation: R Montironi, R Mazzucchelli, \\ A Cimadamore; (VI) Manuscript writing: All authors; (VII) Final approval of manuscript: All authors. \\ Correspondence to: Rodolfo Montironi. Pathological Anatomy, Polytechnic University of the Marche Region, School of Medicine, United Hospitals, \\ Via Conca 71, I-60126 Ancona, Italy. Email: r.montironi@univpm.it.
}

\begin{abstract}
The Gleason grading system, proposed by Dr. Donald F. Gleason in 1966, is one of the most important prognostic factors in men with prostate cancer (PCa). At consensus conferences held in 2005 and 2014, organized by the International Society of Urological Pathology (ISUP), the system was modified to reflect the current diagnostic and therapeutic approaches. In particular, in the 2014 Conference, it was recognized that there were weaknesses with the original and the 2005 ISUP modified Gleason systems. Based on the results of a research conducted by Prof. JI Epstein and his group, a new grading system was proposed by the ISUP in order to address some of such deficiencies: i.e., the five distinct Grade Groups (GGs). Since 2014, results of studies have been published by different groups and societies, including the Genitourinary Pathology Society (GUPS), giving additional support to the prognostic role of the architectural Gleason patterns and, in particular, of the GGs. A revised GG system, taking into account the percentage of Gleason pattern (GP) 4, cribriform and intraductal carcinoma, tertiary GP 5, and reactive stroma grade, has shown to have some advantages, however not ready for adoption in the current practice. The aim of this contribution was to review the major updates and recommendations regarding the GPs and GSs, as well as the GGs, trying to give an answer to the following questions: "How has the grade group system been used in the routine?" and "will the Gleason scoring system be replace by the grade groups?" We also discussed the potential implementation in the future of molecular pathology and artificial intelligence in grading to further define risk groups in patients with PCa.
\end{abstract}

Keywords: Prostate cancer (PCa); Gleason grading; International Society of Urological Pathology (ISUP); 2005 ISUP Gleason modified system; 2014 ISUP Gleason modified system; Genitourinary Pathology Society (GUPS); prognostic grade grouping

Submitted Apr 08, 2020. Accepted for publication Jul 11, 2020.

doi: $10.21037 /$ tau-20-853

View this article at: http://dx.doi.org/10.21037/tau-20-853 


\section{Introduction}

Treatment planning of patients with $\mathrm{PCa}$ depends on the pathological and clinical characteristic predicting the natural course of the tumor. The calculation of the risk of systemic and/or local recurrence following definitive therapy is derived from information obtained from prostate core or/and radical prostatectomy (RP). In this context, $\mathrm{PCa}$ grading is among the most important factors from the prognostic point of view $(1,2)$.

The Gleason grading system, created in 1966, is based on the architecture of $\mathrm{PCa}$ (3). The approach with this system was that, instead of using the worst grade as the grade of the $\mathrm{PCa}$, the grade was the sum of the primary (or predominant) pattern and the secondary (or second most predominant) pattern, the sum being reported as Gleason score (GS) (3). The Gleason system has been adopted for more than 50 years.

At consensus conferences in held 2005 and 2014, organized by the ISUP, the grading system went through several changes in order to reflect in a more accurate manner current therapeutic and diagnostic approaches $(4,5)$. In particular, in the 2005 Consensus Conference, the discussion dealt mainly with controversies related to the grading system previously proposed by Dr. Gleason. The results became known as 2005 ISUP modified Gleason system (4). In the 2014 Consensus Conference, both pathologists and clinicians recognized that there were still weaknesses with the Gleason scoring system (5). Based on the results of a research conducted by Prof. JI Epstein and his group (6), a new grading system was proposed and endorsed by the ISUP in order to address some of such deficiencies: i.e., the five distinct Grade Groups (GGs). Since 2014 a series of studies have been published by different groups and societies, including the GUPS, lending further support to the clinical role of the architectural patterns and of the systems based on GGs (7-13).

The aim of this contribution is to review the major updates and recommendations regarding the GPs and GSs, as well as the GGs, trying to give an answer to the following questions: "How has the grade group system been used in the routine?" and "Will the Gleason scoring system be replace by the grade groups?" It is outside the scope of this contribution to report all the details of the papers published on this topic. The interested reader should consult the original publications. A PubMed search using the keywords "prostate cancer", "Gleason score", "Gleason pattern", "Grade Group", from 2005 and April 2020 was performed.
We present the following article in accordance with the NARRATIVE REVIEW reporting checklist (available at http://dx.doi.org/10.21037/tau-20-853).

\section{Prostate cancer (PCa) grading in 2005: Gleason patterns and scores according to ISUP}

The 2005 ISUP modified Gleason system was proposed by a group of more than 70 urologic pathologists, internationally well known, gathered at the time of the 2005 USCAP meeting in San Antonio, TX. It was based on personal and institutional experience, when there was scant data on the optimal approach, and on data, when available. The major differences between the original PCa grading system proposed by Dr. Gleason and the 2005 ISUP modified Gleason system are in Table 1. From the practical point of view, the major consequence was that the GP 3, in the past the most commonly used, became less used than GP 4 (14-16).

Here is a brief summary of the main updates adopted in $2005(1,4)$ :

* "Individual cells are not be allowed in pattern 3" $(1,4)$;

* "Most of cribriform glands are considered as pattern 4 with only rare cribriform glands meeting the criteria for cribriform pattern 3 " $(1,4)$;

* "The variations of acinar PCa should be graded on the basis of the underlying architecture" $(1,4)$;

* "Ductal PCa is considered as pattern 4, comedonecrosis being pattern 5 and PIN-like ductal adenocarcinoma pattern 3 . There was no consensus on the way mucinous PCa should be scored. When considering sarcomatoid carcinoma, a grade is assigned only to the glandular component, whereas carcinoma with neuroendocrine features is not graded" $(1,4)$;

* "A score of $1+1=2$ is not to be made, with very rare exception. A Gleason score 2-4 on needle cores should be reported rarely, if ever" $(1,4)$;

* "Percent pattern 4-5. It was considered an option to include such information in addition to the Gleason score" $(1,4)$;

* "Reporting secondary patterns of lower grade when present to a limited extent. In the setting of highgrade cancer, one should ignore lower grade patterns if they occupy less than $5 \%$ of the tumour area" $(1,4)$;

* "Reporting secondary patterns of higher grade when present to a limited extent. High-grade tumour of 


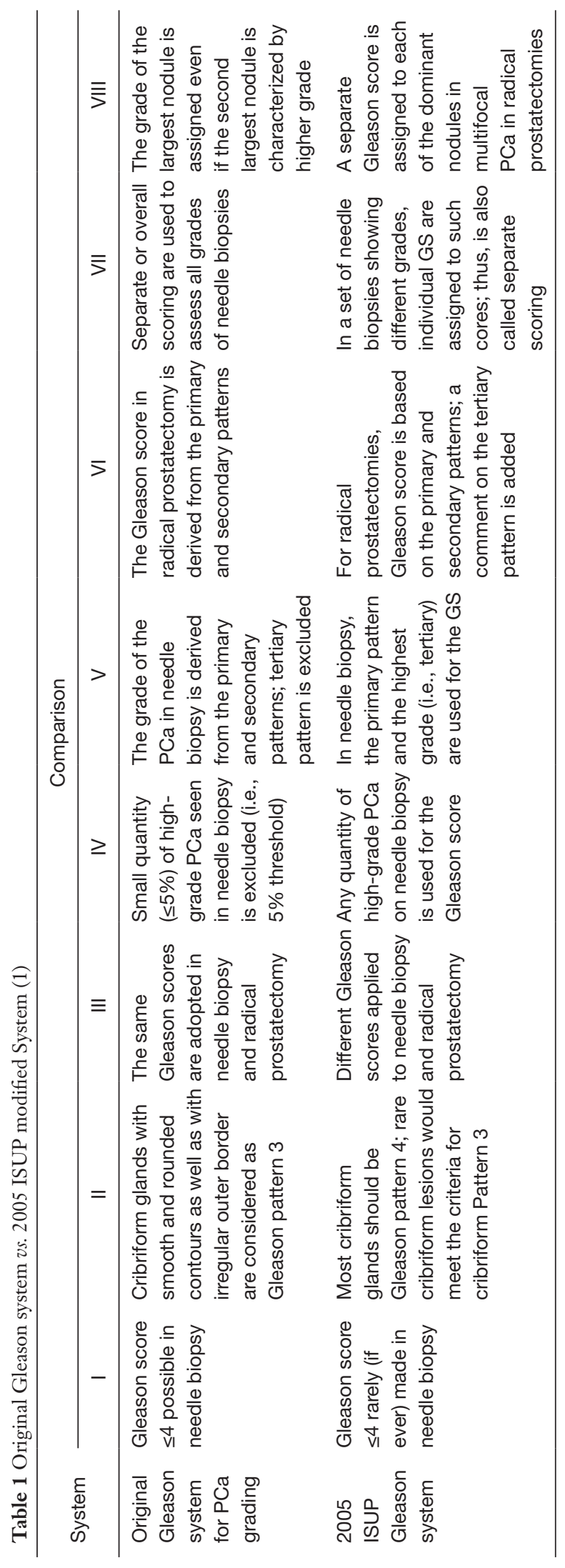

any quantity on needle biopsy should be included within the Gleason score" $(1,4)$;

* On core biopsies with three patterns 3, 4, and 5 (Tertiary pattern), for instance, the primary and the highest patterns are used in the GS. In RP specimens, the GS is derived from the primary and secondary patterns, with a comment on presence of a tertiary pattern" $(1,4)$.

\section{Architectural patterns of PCa: 2014 refinements and definitions by ISUP}

In November 2014, 65 uropathologists, together with 17 urologists, medical oncologists and radiation oncologists, gathered in Chicago, for the 2014 ISUP Consensus Conference in order to update the PCa grading system previously revised in 2005. Updates were based on data from the literature and prognostic validation publications. Table 2 lists the main conclusions reached in the conference. A detailed update of the morphologies of GPs is shown in Table 3. The scores based on such features are also called 2014 ISUP modified GSs. A detailed definition of the GP 4 morphologies follows here $(1,5,16)$ :

* Ill-defined (or poorly formed) glands comprises "glands with poorly formed or absent glandular lumina" (Figure 1A) $(1,5,16)$;

* Fused glands include "fused well- and poorly formed glands" (Figure 1B) $(1,5,16)$;

* Glomeruloid glands are defined as the presence of "dilated glands containing a cribriform proliferation that is attached to only one edge of the gland, resulting in the structure resembling a glomerulus" (Figure 1C) $(1,5,16)$;

* Cribriform is defined as a "solid proliferation with multiple punched-out lumina, without intervening stroma" (Figure 1D). To distinguish small foci of cribriform pattern from fused glands two criteria can be adopted: "Contact of the majority of tumor cells with adjacent stroma (1) and more linear orientation of lumina instead of rounded lumina (2) are both in favor for fused glands" $(1,5,16)$. Figure $1 E$ shows a typical case of PCa with Gleason pattern 3 for comparison.

\section{PCa grade groups: proposed by Jl. Epstein and adopted by ISUP in 2014 and updated in 2017}

\section{Deficiencies with the Gleason scoring system}

The GSs range from 2 to 10 , however, 6 is the lowest 
Table 2 Major conclusions at the 2014 conference

All cribriform glands, either with irregular outer border or with smooth and rounded contour, are considered as Gleason pattern 4

All glomeruloid glands are considered as Gleason pattern 4

Grading of mucinous PCa is based on its underlying growth architecture

Intraductal carcinoma of the prostate is not assigned a grade; a comment as to its association with aggressive PCa is added

Table 3 Gleason patterns: update on their morphologies

Gleason pattern 4 includes the following four morphologies: cribriform, glomeruloid, poorly formed and fused glands

Occasional/seemingly poorly formed or fused glands between well-formed glands is enough for a diagnosis of pattern 4

In those cases that are borderline between pattern 3 and pattern 4 , the lower grade is favored

Branched glands are Gleason pattern 3

Small solid cylinders are Gleason pattern 5

Solid medium to large nests with rosette-like spaces are considered as pattern 5

Presence of unequivocal comedonecrosis is indicative of Gleason pattern 5

usually used. When men are informed that they have a cancer with a GS 6 out of 10, it implies that prognosis should be intermediate between the two extremes, contributing to their idea of harboring a potentially aggressive form of PCa. In addition to this, for therapeutic purposes and in the literature, GSs have been grouped together incorrectly, assuming that they have a similar significance from the prognostic point of view. As an example, several classification systems refer to GS 7, not distinguishing $3+4 v s .4+3$, therefore not considering investigations proving worse clinical outcome for the latter $(5,6,17)$.

\section{Proposal and endorsement of a novel PCa grade groups}

To address some of such limitations, a new grading system was proposed with five distinct GGs, ranging from 1 to 5 (18). This new system was based on a previous study by Epstein and his group (6) and on a validation study of over 20,000 RPs, more than 16,000 core specimens, and more than 5,000 core biopsies before radiation therapy. A summary of morphologic definition of the GGs, already given in details in previous publications, follows here $(1,5,6,19)$ :

* GG 1 (i.e., GS $\leq 6$ ): "Cancers composed only of individual discrete and well-formed glands" $(1,5,6,19)$;
* GG 2 (i.e., GS 3+4=7): “Cancers composed predominantly of discrete and well-formed glands with a lesser component of poorly formed/fused/ glomeruloid/cribriform glands" (1,5,6,19);

* GG 3 (i.e., GS 4+3=7): "Cancers with predominantly poorly formed/fused/glomeruloid/cribriform glands with a lesser component of discrete and well-formed glands" $(1,5,6,19)$;

* GG 4 (i.e., GS 4+4=8; 3+5=8; 5+3=8): "Cancers composed only of poorly formed/fused/glomeruloid/ cribriform glands or tumors with discrete and wellformed glands and lesser component lacking glands, or tumor predominantly lacking glands with a lesser component of discrete and well-formed glands" $(1,5,6,19)$;

* GG 5 (i.e., GS 9 and 10): "Cancers without gland/ luminal formation or with necrosis, with or without poorly formed/fused/glomeruloid/cribriform glands" $(1,5,6,19)$.

Table 4 reports a list of major advantages seen by the participants in the 2014 ISUP conference. The new grades would be used, for the near future, together with the Gleason system, such as GS $3+3=6$ (GG 1), for instance. The GGs 1 to 5 have been endorsed by the World Health Organization (WHO) in the 2016 edition of the blue book Pathology and Genetics: Tumors of the Urinary System and Male Genital Organs", by the College of American 

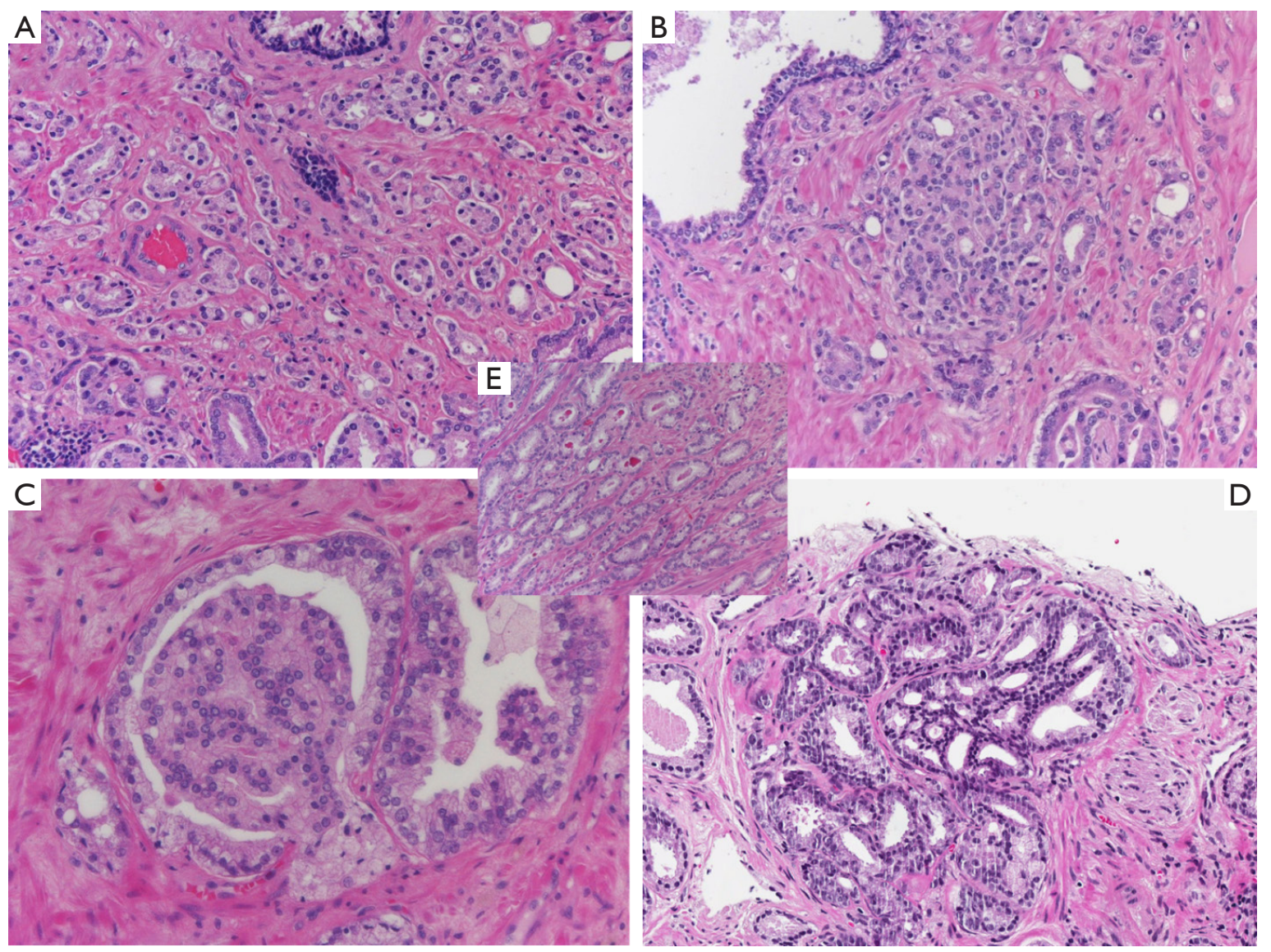

Figure 1 Morphologies of the Gleason pattern 3 and 4: Gleason pattern 4 with poorly formed glands (HE). (A) The lesion is composed elongated nests, compressed elongated glands, of glands with rare or no lumen $(\times 10)$; (B) Gleason pattern 4 with fused glands. The lesion is composed of group of glands that are not completely separated $(\times 10)$; (C) Gleason pattern 4 with glomeruloid structures. The lesion is composed of dilated glands with intraluminal cribriform pattern with a single point of attachment to the periphery, similar to a kidney glomerulus ( $\times 20)$; (D) Gleason pattern 4 with cribriform glands. The lesion is composed of acini with solid proliferation and multiple lumina (×10); (E) PCa Gleason pattern $3(\times 10)$.

Pathologists (CAP protocols) in 2017 and by the $8^{\text {th }}$ Edition of AJCC TNM in 2018.

\section{An update with discussion on practical issues to implement the 2014 ISUP consensus conference}

The updates, provided in 2017 and based on studies published after the 2014 ISUP meeting, have been considered important to promote homogeneity and uniformity in reporting of PCa grades as well as in the management of patients with PCa (20). However, a paper published by Dr. Epstein et al. in 2019, and based on 1576 articles indexed in 2016 to 2017, showed a wide variation in how GSs are grouped (19).
Only two major updates are mentioned specifically here because of their importance for the discussion in the sections that will follow.

\section{Cribriform morphology and grade groups}

An emerging and important issue in studies published after the 2014 conference was that the cribriform morphology is associated with a prognosis that is worse than poorly formed, glomeruloid or fused glands, and should be incorporated into the grading practice (21-32).

Concerning the prostate biopsies, the presence of a cribriform morphology is associated with increased upstaging, upgrading, and positive surgical margins when the surgical specimen is examined (22,31). Flood et al. (23) 
Table 4 Major advantages with the grade grouping system

The grade grouping system provides more precise stratification of cancers patients than the Gleason scoring system

The grade grouping system simplifies the number of categories from Gleason scores 2 to 10 to Grade Groups 1 to 5

The lowest grade in the grade grouping system is 1 not 6 as in Gleason system, the potential being to avoid overtreatment of nonaggressive $\mathrm{PCa}$

demonstrated that perineural invasion on prostate cores, i.e., a feature predicting adverse pathological morphology, such as extraprostatic extension, was "inferior to cribriform morphology in the prediction of non-organ confined PCa" at the time of RP. Kweldam et al. (29) demonstrated that in prostate biopsy the cancer-specific survival of tumors with GS 3+4 (GG 2) without a cribriform pattern was similar to that observed with GS $3+3$ (i.e., GG 1) tumors, being worse in patients with any amount of cribriform pattern.

Concerning the RPs, Dong et al. (21) demonstrated that, at a follow-up of 10 years, $13 \%$ of men with cribriform pattern developed secondary deposits, in comparison with $2.6 \%$ only with GP 4 and no cribriform architecture. Kryvenko et al. (27) showed that patients with metastases in the regional lymph nodes had higher volumes of the cribriform architecture in the RP specimen. The occurrence of a cribriform pattern was linked to a higher biochemical recurrence $(26,28,32)$. The cribriform morphology in a PCa with a GS 8 showed to have a predictive value in terms of cancer specific survival (25).

The GGs should be further updated and include the specific type of GP 4 architecture. It could be, for instance, GS 7 (3+4), "Grade Group 2 cr", to indicate the presence of a specific type GP morphology, while retaining in the pathology report the percentage of GP 4. To indicate the presence of a cribriform architecture and/or of intraductal carcinoma of the prostate (IDC-P; See below), it has been suggested the use of the suffix "C", such as GG 2C, GG 3C, and GG 4C $(16,33)$.

\section{Minor high grade and grade groups}

The agreement in the 2014 ISUP meeting was to substitute the term "tertiary grade pattern" with "minor high-grade pattern". "Minor high grade indicates that the term tertiary should not merely be just the third most common pattern but that it should be minor or limited in extent" (18). A cutoff of $5 \%$ was not dealt with at the time of the 2014 meeting. However, the only threshold of minor high-grade pattern supported by evidence-based data and correlated with prognosis has been the cutoff of $5 \%$.

Concerning the prostate biopsies, the agreement at the 2014 Conference was that the grading rule proposed previously in the 2005 be adopted, i.e., that the most common and highest-grade Gleason patterns are summed to give the GS and that the tertiary pattern is not considered.

Concerning the RP specimens, the term tertiary or minor high-grade pattern are allowed only when there are three patterns, such as with $3+4=7$ (GG 2) with $<5 \%$ GP 5 . The minor high-grade pattern does not change the GG, as it happens in the biopsies. As an example, the pathologist reports GS 3+4=7 (GG 2) with minor (tertiary) pattern 5. At the 2014 Conference the participants discussed how minor high-grade patterns would be handled if the GGs 1 to 5 were to replace GSs. In the example given above, it could be GG 2 with minor high-grade pattern. This could be abbreviated to GG $2+(2,18)$.

\section{Grading patterns and grade groups following 2017: current updates and recommendations}

Controversial issues in Gleason and International Society of Urological Pathology (ISUP) PCa grading: proposed recommendations for international implementation

A paper recently published by Prof. Srigley et al. in the journal Pathology dealt with controversial issues and topics related to PCa grading following the 2014 conference and to propose recommendations for worldwide implementation (34).

The background for this interesting paper was that, while progresses were made at the conference, there were some issues not discussed and/or not resolved, according to the authors. "Most of these items relate to details of assignment of GS and ISUP grade in specific specimen types and grading scenarios", in particular (34):

* Percentage GP 4 in GS 7 tumors;

* Percentage GPs 4 and 5 or 4/5 in GS 8-10 cancer;

* Minor ( $\leq 5 \%)$ high grade patterns when either 2 or 3 Gleason patterns are seen; 
* "Level of reporting (core, specimen, case), dealing with grade diversity among site (highest and composite scores) and reporting scores in radical prostatectomy specimens with multifocal disease" (34).

The recommendations, mostly based on consolidated expert opinion, were classified as established if there was prior agreement by consensus and provisional if there was no previous agreement or not discussed in a previous consensus conference. For some issues, reporting options reflecting the local requirements and diverse practice models were recommended.

The recommendations have provided a basis for discussion in future meetings, such as the ISUP PCa Grading Conference in Nice, France, of early September 2019.

According to the ISUP Newsletter October 2019, "several grading-related issues remaining after the ISUP 2014 conference in Chicago were resolved, such as for instance the inclusion of minor grades in the ISUP grade of prostatectomy specimens, the reporting of intraductal and cribriform pattern carcinoma and its incorporation in grading, the reporting of grade in MRI targeted biopsies and the reporting of benign findings in false negative (PIRADS 4/5) prostate biopsies."

A manuscript outlining the consensus statements on PCa grading, based on the pre-meeting survey and the conference in Nice, not yet available to the authors at the time of writing this review, should appear soon (Supplementary file).

\section{Intraductal carcinoma of the prostate is an aggressive form of invasive carcinoma and should be graded}

Prof. Samaratunga et al. (35) have published a paper on IDC-P in the February issue of the journal Pathology. The authors observe that retrograde invasion of the prostatic ducts and acini by an invasive PCa occurs with a certain frequency. It is usually associated with high grade and high stage disease as well as with poor prognosis. This is linked to its morphologic, immunohistochemical, and molecular features.

Gleason did not distinguish between IDC-P and invasive $\mathrm{PCa}$ when he developed and validated his system. The recommendation of the WHO is that IDC-P should not be considered when grading PCa. Recent studies have suggested that the incorporation of IDC-P into the grading of the tissue specimen can provide additional clinical information with prognostic significance (35-38).

\section{The 2019 Genitourinary Pathology Society (GUPS) white paper on contemporary grading of PCa}

Members of the GUPS have a paper on this topic in the journal Archives of Patbology and Laboratory Medicine (still in press at the time of writing this contrition, however made widely available).

For the readers that are not yet familial, GUPS is "a society formed in 2017 as a global organization of uropathologists with a vision to advance the care of patients with urologic diseases through improvements in the subspecialty of urological pathology by enhancing best practices, research, and education. The paper is based on GUPS surveys on practice patterns relating to grading were formulated and sent to both GUPS pathology members as well as to several clinician groups" (13).

The authors of the paper presented the first PCa grading recommendations from the GUPS, addressing key issues and areas concerning:

* Grading of cribriform cancer and recording extent of GP 4;

* Grading cores versus the specimens, especially with respect to reporting multiparametric MRI targeted biopsies;

* Minor tertiary/minor high grades;

* Reporting grading intraductal cancer;

* "The issue of whether immunohistochemistry has any role in accurate grading is prescient";

* "With a continued eye on the future, there is an update on two key areas of molecular pathology and artificial intelligence in grading" (13) (Supplementary file).

\section{Gleason patterns and grade groups: potential steps forward}

\section{Grade groups supplemented with molecular testing}

Our understanding of the molecular data in PCa in the context of the disease as well as complement to the grading system continues to show promise $(13,29)$.

Genomic support for GGs based on whole exome and whole genome sequencing information from localized PCa was evaluated by Rubin et al. They showed an increased observation of genomic amplifications and deletions associated with increasing risk strata. They also showed 
increased non-synonymous point mutations. GG 1 was haploid, whereas GGs 2 to 5 were characterized by an increasing frequency of polyploidy. Principal Component Analysis identified distinct profiles between the 5 GGs, i.e., GG 1 through GG 3 as separate groups, but showing similarity, from the genomic point of view, for GG 4 and GG 5 (39).

Germline and somatic testing for mutations in DNA repair pathway genes is nowadays recommended in high risk patients with clinically localized $\mathrm{PCa}$, including those with GG 4 or 5 cancers (39).

\section{Grade groups supplemented with quantitative methods}

There are studies on quantitative methods on how to detect PCa and automate PCa diagnosis and grading, and therefore the GGs (40). A study by Diamond et al. (41) used image analysis of prostate tissue abnormalities in tissue sections. They developed a machine vision system that "facilitated the potential of quantitative methods to provide highly discriminatory information in the automated identification of prostatic lesions". Their system was able of identifying $\mathrm{PCa}$, including the identification of cribriform gland with a cribriformity index $(40,41)$. A more recent study has reported "the successful identification of visually meaningful histopathological features for automatic grading of PCa” (42). Additional studies are being published on this topic.

Commercially available tools for PCa detection and grading are starting to become available in the market. In the next 3 to 5 years there will be a number of robust clinical grade products to supplement the morphologic diagnosis and contribute to create more "automated" and "standardized" approaches to grade PCa (13). Artificial intelligence has the opportunity to empower pathologists with greater impact on the care of patients (13).

\section{Grade groups supplemented with nuclear morphology}

The Gleason system, including the GGs, is based on the architecture of cancer. The contribution of nuclear features to the enhance the prognostic significance of the GSs and GGs has been analyzed occasionally. "It is worth mentioning the proposal made by Dr. Mostofi in a 1999 WHOsponsored meeting, to supplement the Gleason grading system with the WHO nuclear grading scheme" (43). Patients with GS 3+3=6 (i.e., GG 1) and WHO nuclear grade 3 show higher cancer specific mortality compared to those with lower nuclear grades (43). This was confirmed in a karyometric investigation showing that the nuclear signature is great important to further define risk groups in patients with $\mathrm{PCa}$ (44).

\section{Conclusions}

In 2014 conference there was an overwhelming approval for the adoption in the routine of the 5 GG system, based on the GPs $(5,18)$. It was also passed the proposal that the GGs should be reported together with the 2014 ISUP GSs, the idea being in the future the GGs only will be used.

Papers published after the 2014 conference have shown that the new PCa grading system has several advantages, such as:

* More precise grade stratification than the 2014 ISUP modified GSs;

* Simplified system of five grades only, the lowest being 1 , as opposed to 6 ; and

* Potential to reduce the risk of overtreatment of patients with PCa.

The times in which the GG system based on the GPs is used as an alternative to the GSs are mature (8).

Novel approaches and refinements of the GGs, factoring in the percent GP 4, tertiary GP 5, cribriform carcinoma, IDC-P as well as reactive stroma grade, have been proposed and have several practical advantages, but are not considered ready for adoption in the current practice $(1,2,13,16,45)$.

\section{Acknowledgments}

Funding: None.

\section{Footnote}

Provenance and Peer Review: This article was commissioned by the Guest Editors (Rodolfo Montironi, Alessia Cimadamore, Antonio Lopez-Beltran, Marina Scarpelli and Liang Cheng) for the series "Update on Molecular Classification and Individualized Treatments of Genitourinary Tumors" published in Translational Andrology and Urology. The article was sent for external peer review organized by the Guest Editor and the editorial office.

Reporting Checklist: The authors have completed the Narrative Review reporting checklist. Available at http:// 
dx.doi.org/10.21037/tau-20-853

Conflicts of Interest: All authors have completed the ICMJE uniform disclosure form (available at http:// dx.doi.org/10.21037/tau-20-853). The series "Update on Molecular Classification and Individualized Treatments of Genitourinary Tumors" was commissioned by the editorial office without any funding or sponsorship. RM, LC, AC, MS, and ALB served as the unpaid Guest Editor of the series. LC serves as an unpaid editorial board member of Translational Andrology and Urology from Dec 2018 to Nov 2022. The authors have no conflicts of interest to declare.

Ethical Statement: The authors are accountable for all aspects of the work in ensuring that questions related to the accuracy or integrity of any part of the work are appropriately investigated and resolved.

Open Access Statement: This is an Open Access article distributed in accordance with the Creative Commons Attribution-NonCommercial-NoDerivs 4.0 International License (CC BY-NC-ND 4.0), which permits the noncommercial replication and distribution of the article with the strict proviso that no changes or edits are made and the original work is properly cited (including links to both the formal publication through the relevant DOI and the license). See: https://creativecommons.org/licenses/by-nc-nd/4.0/.

\section{References}

1. Montironi R, Santoni M, Mazzucchelli R, et al. Prostate cancer: from Gleason scoring to prognostic grade grouping. Expert Rev Anticancer Ther 2016;16:433-40.

2. Magi-Galluzzi C, Montironi R, Epstein JI. Contemporary Gleason grading and novel Grade Groups in clinical practice. Curr Opin Urol 2016;26:488-92.

3. Gleason DF. Classification of prostatic carcinomas. Cancer Chemother Rep 1966;50:125-28.

4. Epstein JI, Allsbrook WC Jr, Amin MB, Egevad LL, et al. The 2005 International Society of Urological Pathology (ISUP) Consensus Conference on Gleason Grading of Prostatic Carcinoma. Am J Surg Pathol 2005;29:1228-42.

5. Epstein JI, Egevad L, Amin MB, et al. The 2014 international society of urological pathology (ISUP) consensus conference on Gleason grading of prostatic carcinoma: definition of grading patterns and proposal for a new grading system. Am J Surg Pathol 2016;40:244-52.
6. Pierorazio PM, Walsh PC, Partin AW, et al. Prognostic Gleason grade grouping: data based on the modified Gleason scoring system. BJU Int 2013;111:753-60.

7. Delahunt B, Egevad L, Srigley JR, et al. Validation of international society of urological pathology (ISUP) grading for prostatic adenocarcinoma in thin core biopsies using TROG 03.04 'RADAR' trial clinical data. Pathology 2015;47:520-25.

8. Epstein JI, Zelefsky MJ, Sjoberg DD, et al. A contemporary prostate cancer grading system: a validated alternative to the Gleason score. Eur Urol 2016;69:428-35.

9. Erickson A, Sandeman K, Lahdensuo K, et al. New prostate cancer grade grouping system predicts survival after radical prostatectomy. Hum Pathol 2018;75:159-66.

10. Loeb S, Folkvaljon Y, Robinson D, et al. Evaluation of the 2015 Gleason grade groups in a nationwide populationbased cohort. Eur Urol 2016;69:1135-41.

11. Samaratunga H, Delahunt B, Gianduzzo T, et al. The prognostic significance of the 2014 International Society of Urological Pathology (ISUP) grading system for prostate cancer. Pathology 2015;47:515-19.

12. Spratt DE, Cole AI, Palapattu GS, et al. Independent surgical validation of the new prostate cancer gradegrouping system. BJU Int 2016;118:763-69.

13. Trpkov K, Werneck Cunha I, Epstein JI, et al. The 2019 Genitourinary Pathology Society (GUPS) white paper on contemporary grading of prostate cancer. Arch Pathol Lab Med 2020. [Epub ahead of print].

14. Egevad L, Mazzucchelli R, Montironi R. Implications of the international society of urological pathology modified Gleason grading system. Arch Pathol Lab Med 2012;136:426-34.

15. Montironi R, Cimadamore A, Cheng L, et al. Prostate cancer grading in 2018: limitations, implementations, cribriform morphology, and biological markers. Int J Biol Markers 2018;33:331-34.

16. Montironi R, Cimadamore A, Gasparrini S, et al. Prostate cancer with cribriform morphology: diagnosis, aggressiveness, molecular pathology and possible relationships with intraductal carcinoma. Expert Rev Anticancer Ther 2018;18:685-93.

17. Esserman LJ, Thompson IM, Reid B, et al. Addressing overdiagnosis and overtreatment in cancer: a prescription for change. Lancet Oncol 2014;15:e234-42.

18. Epstein JI, Amin MB, Reuter VE, et al. Contemporary Gleason grading of prostatic carcinoma: an update with discussion on practical issues to implement the 2014 
international society of urological pathology (ISUP) consensus conference on Gleason grading of prostatic parcinoma. Am J Surg Pathol 2017;41:e1-7.

19. Montironi R, Cheng L, Scarpelli M, et al. From Gleason grading system and highgrade tertiary patterns to grade groups and integrated quantitative Gleason score. Eur Urol 2018;73:684-6.

20. Zhou AG, Salles DC, Samarska IV, et al. How are Gleason scores categorized in the current literature: An analysis and comparison of articles published in 2016-2017. Eur Urol 2019;75:25-31.

21. Dong F, Yang P, Wang C, et al. Architectural heterogeneity and cribriform pattern predict adverse clinical outcome for Gleason grade 4 prostatic adenocarcinoma. Am J Surg Pathol 2013;37:1855-61.

22. Flood TA, Schieda N, Keefe DT, et al. Utility of Gleason pattern 4 morphologies detected on transrectal ultrasound (TRUS)-guided biopsies for prediction of upgrading or upstaging in Gleason score $3+4=7$ prostate cancer. Virchows Arch 2016;469:313-9.

23. Flood TA, Schieda N, Keefe DT, et al. Perineural invasion on biopsy is associated with upstaging at radical prostatectomy in Gleason score $3+4=7$ prostate cancer. Pathol Int 2016;66:629-32.

24. Haffner MC, Salles DC, Gao G, et al. Gleason pattern 4 with cribriform morphology on biopsy is associated with adverse clinicopathological findings in a prospective radical prostatectomy cohort. Hum Pathol 2020;98:74-80.

25. Harding-Jackson N, Kryvenko ON, Whittington EE, et al. Outcome of Gleason $3+5=8$ prostate cancer diagnosed on needle biopsy: prognostic comparison with Gleason $4+$ $4=8$. J Urol 2016;196:1076-81.

26. Kir G, Sarbay BC, Gümüş E, et al. The association of the cribriform pattern with outcome for prostatic adenocarcinomas. Pathol Res Pract 2014;210:640-4.

27. Kryvenko ON, Gupta NS, Virani N, et al. Gleason score 7 adenocarcinoma of the prostate with lymph node metastases: analysis of 184 radical prostatectomy specimens. Arch Pathol Lab Med 2013;137:610-7.

28. $\mathrm{Ku} \mathrm{JY}$, Lee $\mathrm{CH}, \mathrm{Kim} \mathrm{KH}$, et al. Cribriform pattern is risk factor of biochemical recurrence in positive surgical margin patients. Eur Urol Suppl 2017;16:e2675.

29. Kweldam CF, Kümmerlin IP, Nieboer D, et al. Prostate cancer outcomes of men with biopsy Gleason score 6 and 7 without cribriform or intraductal carcinoma. Eur J Cancer 2016;66:26-33.

30. Kweldam CF, Wildhagen MF, Steyerberg EW, et al. Cribriform growth is highly predictive for postoperative metastasis and disease-specific death in Gleason score 7 prostate cancer. Mod Pathol 2015;28:457-64.

31. Sarbay BC, Kir G, Topal CS, et al. Significance of the cribriform pattern in prostatic adenocarcinomas. Pathol Res Pract 2014;210:554-7.

32. Trudel D, Downes MR, Sykes J, et al. Prognostic impact of intraductal carcinoma and large cribriform carcinoma architecture after prostatectomy in a contemporary cohort. Eur J Cancer 2014;50:1610-6.

33. Iczkowski KA, Paner GP, Van der Kwast T. The new realization about cribriform prostate cancer. Adv Anat Pathol 2018;25:31-7.

34. Srigley JR, Delahunt B, Samaratunga $\mathrm{H}$, et al. Controversial issues in Gleason and international society of urological pathology (ISUP) prostate cancer grading: proposed recommendations for international implementation. Pathology 2019;51:463-73.

35. Samaratunga H, Delahunt B, Egevad L, et al. Intraductal carcinoma of the prostate is an aggressive form of invasive carcinoma and should be graded. Pathology 2020;52:192-6.

36. Epstein JI. Is There enough support for a new prostate grading system factoring in intraductal carcinoma and cribriform cancer? Eur Urol 2020;77:199-200.

37. Kweldam CF, Kümmerlin IP, Nieboer D, et al. Presence of invasive cribriform or intraductal growth at biopsy outperforms percentage grade 4 in predicting outcome of Gleason score 3+4=7 prostate cancer. Mod Pathol 2017;30:1126-32.

38. van Leenders GJLH, Kweldam CF, Hollemans E, et al. Improved prostate cancer biopsy grading by incorporation of invasive cribriform and intraductal carcinoma in the 2014 Grade Groups. Eur Urol 2020;77:191-8.

39. Rubin MA, Girelli G, Demichelis F. Genomic correlates to the newly proposed grading prognostic groups for prostate cancer. Eur Urol 2016;69:557-60.

40. Montironi R, Cheng L, Lopez-Beltran A, et al. Quantitative image analysis on histologic virtual slides for prostate pathology diagnosis, response to chemopreventive agents, and prognosis. Eur Urol Focus 2017;3:467-9

41. Diamond J, Anderson NH, Bartels PH, et al. The use of morphological characteristics and texture analysis in the identification of tissue composition in prostatic neoplasia. Hum Pathol 2004;35:1121-31

42. Niazi MKK, Keluo Y, Zynger DL, et al. Visually meaningful histopathological features for automatic grading of prostate cancer. IEEE J Biomed Health Inform 2017;21:1027-38. 
43. Montironi R, Scarpelli M, Mazzucchelli R, et al. Does prostate acinar adenocarcinoma with Gleason Score $3+3=6$ have the potential to metastasize? Diagn Pathol 2014;9:190.

44. Venkataraman G, Rycyna K, Rabanser A, et al.

Morphometric signature differences in nuclei of Gleason

Cite this article as: Montironi R, Cheng L, Cimadamore A, Mazzucchelli R, Scarpelli M, Santoni M, Massari F, Lopez-Beltran A. Narrative review of prostate cancer grading systems: will the Gleason Scores be replaced by the Grade Groups? Transl Androl Urol 2021;10(3):1530-1540. doi: 10.21037/tau-20-853 pattern 4 areas in Gleason 7 prostate cancer with differing primary grades on needle biopsy. J Urol 2009;181:88-93.

45. Hollemans E, Verhoef EI, Bangma CH, et al. Large cribriform growth pattern identifies ISUP grade 2 prostate cancer at high risk for recurrence and metastasis. Mod Pathol. 2019;32:139-46. 
After the submission of this manuscript, the following paper has been published Online ahead of print by van Leenders GJLH et al. The 2019 International Society of Urological Pathology (ISUP) Consensus Conference on Grading of Prostatic Carcinoma (46).

From the Abstract of the paper by van Leenders et al: "The manuscript summarizes the proceedings of the ISUP consensus meeting for grading of prostatic carcinoma held in September 2019, in Nice, France. Topics brought to consensus included the following: (I) approaches to reporting of Gleason patterns 4 and 5 quantities, and minor/ tertiary patterns, (II) an agreement to report the presence of invasive cribriform carcinoma, (III) an agreement to incorporate intraductal carcinoma into grading, and (IV) individual versus aggregate grading of systematic and multiparametric magnetic resonance imaging-targeted biopsies. Finally, developments in the field of artificial intelligence in the grading of prostatic carcinoma and future research perspectives were discussed".

\section{References}

46. van Leenders GJLH, van der Kwast TH, Grignon DJ, et al. The 2019 International Society of Urological Pathology (ISUP) Consensus Conference on Grading of Prostatic Carcinoma. Am J Surg Pathol 2020;44:e87-99. 